\title{
Combinatorial Yamabe Flow on Surfaces
}

\section{Feng Luo}

\begin{abstract}
In this paper we develop an approach to conformal geometry of piecewise flat metrics on manifolds. In particular, we formulate the combinatorial Yamabe problem for piecewise flat metrics. In the case of surfaces, we define the combinatorial Yamabe flow on the space of all piecewise flat metrics associated to a triangulated surface. We show that the flow either develops removable singularities or converges exponentially fast to a constant combinatorial curvature metric. If the singularity develops, we show that the singularity is always removable by a surgery procedure on the triangulation. We conjecture that after finitely many such surgery changes on the triangulation, the flow converges to the constant combinatorial curvature metric as time approaches infinity.
\end{abstract}

\section{$\S 1$. Introduction}

1.1. In this paper we consider the class of piecewise flat metrics on a triangulated manifold and develop an approach to piecewise linear (PL) conformal geometry of these metrics. We also formulate the combinatorial Yamabe problem for piecewise flat metrics. The notion of conformal class of a metric was originated from Riemannian metrics. Given a Riemannian metric $g_{i j}$ on a smooth closed manifold, the conformal change of the metric is a new Riemannian metric of the form $u g_{i j}$ where $u$ is some smooth positive function defined on the manifold. The Yamabe problem states that there exists a constant scalar curvature metric in the conformal class of any Riemannian metric. This was solved affirmatively for closed Riemannian manifolds in [Ya], [Tu], [Au] and [Sc]. The solution was considered as a milestone in application of non-linear partial differential equations in geometry. In trying to develop the analogous PL conformal geometry, one faces the task of defining the notion of PL conformal class of a PL metric. The most naive approach is to take a local (infinitesimal) point of view. Namely, since the conformality in smooth category means infinitesimal invariance of angles, thus PL conformal geometry should preserve the of measurment of angles in the PL metrics even at the singularities. However, we are not able to come up with any reasonable results from this point of view. It is probably due to the that fact that local geometry of smooth metrics is governed by linear algebra and the local geometry of PL metric is governed by the geometry of simplexes. We propose to approach the analogous PL conformal geometry of PL metrics from global point of view in this paper. Namely, the conformal class of a smooth metric comes from the action of smooth functions on smooth metrics. Thus the PL conformal class of a PL metric should be a class of PL metrics related by the action of functions defined on the set of all vertices. Our main results indicate that the PL conformal geometry captures some of the main features of the smooth conformal geometry on surfaces. The situation in PL theory is more complicated in the sense that there are triangulations on surfaces so that any PL metric associated to the triangulatinos has none constant PL scalar curvature. This is mainly caused by the fact that a PL metric is supported by many different triangulations. 
In the case of surfaces, we prove that the existence of a constant PL scalar curvature metric is a purely topological condition on the triangulation (theorem 1.1). We propose to find the constant PL scalar curvature metric in the PL conformal class by a system of ordinary differential equations. These equations seems to be the right analogy of the Yamabe flow. We establish some of the basic properties of the flow. Especially, we prove that the flow will not develop essential singularities in finite time and converges exponentially fast to the constant PL curvature metric if no singularity develops. The most interesting property of the flow is that when the flow develops singularity, the flow naturally suggests a way to do surgery on the underlying triangulation. This indicates that the flow tends to search for both the best piecewise flat metric and the underlying triangulation.

1.2. We begin with a dictionary between piecewise linear (PL) and smooth theory. In the smooth theory, we start with a smooth manifold $N^{n}$ and whereas in the PL theory, we start with a triangulated manifold $\left(M^{n}, T\right)$ where $T$ is the triangulation. The natural analogy of the smooth functions on $N^{n}$ are the functions defined on the set of all vertices $V$ in the triangulation $T$. Similarly, the analogy of tensors on $N^{n}$ are functions defined on the set of all $i$-simplexes in the triangulation. For instance, the de Rham cohomology and simplicial cohomology are such examples. We define a $P L$ metric associated to the triangulation to be a positive real valued function defined on the set $E$ of all edges (i.e., 1-simplexes) of $T$ so that for each n-simplex in $T$, the restriction of the function to the 1-simplexes of the n-simplex is the edge lengths of some Euclidean n-simplex. The last condition is the realizability condition which is given by the Cayley-Menger matrices for Euclidean n-simplexes. We may think of a PL metric as a metric on the manifold so that its restriction to each simplex is isometric to a Euclidean simplex. Just like the space of all Riemannian metrics on a smooth manifold is convex, it can be shown that the space of all PL metrics associated to a triangulation is a convex set. Indeed, it is an observation of Igor Rivin that the set $\left\{d^{2}: E \rightarrow \mathbf{R} \mid\right.$ where $d: E \rightarrow \mathbf{R}_{>0}$ is a PL metric associated to $T\}$ is convex in $\mathbf{R}^{E}$. For any Riemannian metric, the curvature tensor assigns to every four tangent vectors at a point a number. For the PL metric, there are many notions of curvatures (see $[\mathrm{CMS}],[\mathrm{CE}]$ and others). The most natural curvature seems to be the function $K$ which assigns to each (n-2)-simplex $\sigma_{n-2}$ the number $K\left(\sigma_{n-2}\right)=2 \pi-\alpha$ where $\alpha$ is the sum of all dihedral angles of $n$-simplexes at the (n-2)-simplex. It is shown in [St] that if the $K\left(\sigma_{n-2}\right)>0$ for all (n-2)-simplexes, then the manifold has finite fundamental group. This is the analogy of the Myers' theorem in the smooth theory. The combinatorial scalar curvature (or PL scalar curvature) of a $\mathrm{PL}$ metric $d$ is a function $S_{d}: V \rightarrow \mathbf{R}$ whose value at the vertex $v$ is $S_{d}(v)=\sum_{\sigma_{n-2}>v} K\left(\sigma_{n-2}\right) \operatorname{vol}\left(\sigma_{n-2}\right)$ where the summation is over all $(n-2)$-simplexes having $v$ as a vertex. If $n=2$, then, as a convention, we set $\operatorname{vol}(v)=1$. Note that if $\lambda$ is a positive number then $S_{\lambda d}=\lambda^{n-2} S_{d}$ and also that the total scalar curvature $\sum_{v \in T^{0}} S(v)$ is exactly Regge's proposed approximation to the Einstein action (see $[\mathrm{Re}]$ ). Furthermore, the PL scalar curvature $S_{d}$ depends not only on the metric $d$ (as a metric in point set topology) but also on the choice of the underlying triangulation $T$. If the manifold is a surface, then the scalar curvature $S(v)$ is the curvature $K(v)$.

Using this dictionary, it is now natural to define a $P L$ conformal factor for a PL metric to be a positive function $u$ defined on the set $V$ of all vertices. Given a PL metric 
$d: E \rightarrow \mathbf{R}_{>0}$, we define the PL conformal change of $d$ to be the new metric $u * d: E \rightarrow \mathbf{R}_{>0}$ where $u * d\left(v v^{\prime}\right)=u(v) u\left(v^{\prime}\right) d\left(v v^{\prime}\right)$ and $v v^{\prime}$ is the edge with vertices $v$ and $v^{\prime}$ so that $u * d$ is realizable on each $n$-simplex. The set of all PL metrics of the form $u * d$ for a fixed $d$ is called the PL conformal class of the PL metric $d$.

The combinatorial Yamabe problem asks if there is a constant PL scalar curvature metric within the PL conformal class of any PL metric. As we will see, there are topological obstructions for the existence of the constant scalar curvature PL metrics associated to a triangulation even in dimension 2 (see theorem 1.1). The goal is to establish the existence of constant PL scalar curvature metric when the topological obstructions vanish.

1.3. In the case of a triangulated surface, we propose to approach the combinatorial Yamabe problem using a system of ordinary differential equations. Here is the setup. Let $T$ be a triangulation of a closed surface $M$ and $V$ and $E$ be the set of all vertices and edges in $T$. A PL metric associated to $T$ is a positive function $d: E \rightarrow \mathbf{R}$ so that it satisfies the triangular inequalities on the three edges of any triangle in $T$. Fix the PL metric $d$ on the surface. Let $u: V \rightarrow \mathbf{R}_{>0}$ be a conformal factor. For simplicity, let us write $V=\left\{v_{1}, \ldots, v_{N}\right\}$ and $u_{i}=u\left(v_{i}\right)$. If $v_{i} v_{j}$ is an edge in $T$, then $d\left(v_{i} v_{j}\right)$ is denoted by $d_{i j}$. We define the combinatorial Yamabe flow to be the following system of ordinary differential equations.

$$
\begin{gathered}
\frac{d u_{i}}{d t}=-u_{i} K_{i} \\
u_{i}(0)=1
\end{gathered}
$$

Here the curvature $K_{i}$ is the $K$ curvature of the metric $u * d$ at time $t$ at the i-th vertex $v_{i}$.

The Gauss-Bonnet theorem for PL metrics states that $\sum_{i=1}^{N} K_{i}=2 \pi \chi(M)$. Thus the average curvature $K_{a v}$ is $2 \pi \chi(M) / N$. In particular, the constant combinatorial curvature PL metric has curvature $K_{a v}$ at all vertices. The combinatorial Yamabe problem asks if this metric exists in the PL conformal class.

There is a combinatorial obstruction for the existence of the constant curvature PL metric associated to a triangulation $T$. In fact the following holds. (For a finite set $X$, we use $|X|$ to denote the number of elements in $X$.)

Theorem 1.1. Fix a triangulation $T$ of a closed topological surface $M$. There exists a constant $P L$ scalar curvature metric associated to $T$ if and only if for any proper subset $I$ of the vertices $V$ of $T$,

$$
\left|F_{I}\right| /|I|>|F| /|V|
$$

where $F$ is the set of all triangles in $T$ and $F_{I}$ is the set of all triangles having a vertex in $I$. 
Furthermore, the condition (*) holds for all triangulations of surfaces of non-negative Euler characteristic.

For simplicity, we call a triangulation which supports a constant curvature PL metric admissible.

There are none admissible triangulations on each closed surface of negative Euler characteristic. It can be shown that given any triangulation of a surface, there is a subdivision of it which is admissible. However, we are not sure if the subvision can be choosen to be an iterated barycentric subdivision or even the 2-dimensional subdivision which replace each triangle by four triangles formed by the barycenters of the vertices and edges. On the other hand, if our conjecture in section 1.3 holds, then every triangulation of the surface is equivalent to an admissible one having the same number of vertices.

Our main results concerning the combinatorial Yamabe flow can be summarized in the following theorems.

Theorem 1.2. Fix a piecewise flat metric $d$ on a triangulated surface $(M, T)$. The following holds.

(a). Under the combinatorial Yamabe flow, the curvature evolves according to a combinatorial heat equation of the form

$$
\frac{d K_{i}}{d t}=\sum_{j=1}^{N} c_{i j} K_{j}
$$

where the matrix $\left[c_{i j}\right]_{N \times N}$ is symmetric and semi-negative definite. In particular, the total curvature $\sum_{i=1}^{N} K_{i}(t)^{2}$ is decreasing in time $t$.

(b). The combinatorial Yamabe flow is variational. If we change the variable $u_{i}$ to $w_{i}=\log u_{i}$, then the combinatorial Yamabe flow is the negative gradient flow of a locally convex function in $w=\left(w_{1}, \ldots, w_{N}\right)$.

(c). (local rigidity) The curvature map $K:\left\{u=\left(u_{1}, \ldots, u_{N}\right) \in \mathbf{R}_{>0}^{N} \mid \prod_{i=1}^{N} u_{i}=1, u * d\right.$ is again a $P L$ metric $\} \rightarrow\left\{\left(k_{1}, \ldots, k_{N}\right) \mid \sum_{i=1}^{N} k_{i}=2 \pi \chi(M)\right\}$ sending a conformal factor $u$ to the curvature of the metric $u * d$ is a local homeomorphism.

We do not know if this local rigidity can be improved to be a global rigidity result.

To understand the long time behavior of the flow, we introduce the normalized equation

$$
\begin{gathered}
\frac{d u_{i}}{d t}=-u_{i}\left(K_{i}-K_{a v}\right) \\
u_{i}(0)=1
\end{gathered}
$$


The two equations (1.1) and (1.3) are equivalent in the sense that $u_{i}(t)$ solves (1.1) if and only if $e^{2 \pi \chi(M) t / N} u_{i}(t)$ solves (1.3). Furthermore, if $u(t)=\left(u_{1}(t), \ldots, u_{N}(t)\right)$ is a solution to the normalized equation (1.3) then the product $\prod_{i=1}^{N} u_{i}(t)=1$ for all time $t$. To understand the asymptotic behavior of the solution of (1.3), we have to analysis the potential formation of singularities in the normalized equation (1.3). There are only two types of singularities which may occur. Suppose $u(t)$ is a solution of (1.3) in the time interval $[0, L)$ where $L \leq \infty$. We say the solution develops an essential singularity at time $L$ if there is an index $i$ and a sequence of time $t_{n}$ approaching $L$ so that $\lim _{t_{n} \rightarrow L} u_{i}\left(t_{n}\right)=0$. We say the solution develops removable singularity at time $L$ if there is a sequence of time $t_{n}$ approaching $L$ so that $u_{i}\left(t_{n}\right)$ remains in a compact set in $\mathbf{R}_{>0}$ for all $i$ and there is a triangle $\Delta v_{i} v_{j} v_{k}$ in $T$ which degenerates into a line segment as $t_{n} \rightarrow L$. The last condition means that the triangular inequality $u_{i} d_{i k} u_{k}+u_{k} d_{k j} u_{j}>u_{i} d_{i j} u_{j}$ becomes equality as $t_{n} \rightarrow L$ for some indices $i, j, k$.

Theorem 1.3. (a) For any triangulated surface, the normalized combinatorial Yamabe flow will not develop essential singularity in finite time.

(a) If the triangulation is admissible, the normalized combinatorial Yamabe flow will not develop essential singularity at time infinity.

If removable singularity occurs, say the vertex $v_{k}$ is moving toward the interior of the edge $v_{i} v_{j}$, then we change the original triangulation $T$ as follows. Let $v_{l}$ be the fourth vertex in $T$ so that $v_{i} v_{j} v_{l}$ forms a triangle in $T$. Then we replace the triangulation $T$ by a new triangulation $T^{\prime}$ which is obtained from $T$ by deleting the edge $v_{i} v_{j}$ and adding a new edge $v_{k} v_{l}$. In particular, two triangles $\Delta v_{i} v_{j} v_{k}$ and $\Delta v_{i} v_{j} v_{l}$ in $T$ are replaced by two new triangles $\Delta v_{k} v_{l} v_{i}$ and $\Delta v_{k} v_{l} v_{j}$. This is the most common surgery operation on triangulations of surfaces. After the combinatorial surgery on $T$, we run the combinatorial Yamabe flow on $T^{\prime}$ with initial metric coming from the final stage of metric on $T$ at time $L$. We conjecture that after finitely many such surgery operations on the triangulation $T$, the combinatorial Yamabe flow converges exponentially fast to the constant curvature metric. This is supported by the following.

Theorem 1.4. If no singularity develops in the normalized combinatorial Yamabe flow, then the solution converges exponentially fast to a constant curvature PL metric as time approaches infinity.

Under the normalized flow, the distance function restricted to the 1-skeleton of the triangulation will stay in the same quasi-isometric class in any compact time interval in $\mathbf{R}_{>0}$. The geometry of the quasi-isometry class changes only when the essentialy singulariy develops at time infinity.

If we start with a single Euclidean triangle, and define the curvature $K_{i}$ at the i-th vertex to be $\pi-\theta_{i}$ where $\theta_{i}$ is the inner angle at the $\mathrm{i}$-th vertex, then it can be shown that the corresponding normalized equation (1.3) has solution for all time and converges exponentially fast to the equilateral triangle as time tends to infinity.

The following is likely to hold for the combinatorial Yamabe flows on surfaces. 

class.

Conjecture. (a) A constant PL scalar curvature metric is unique in its $P L$ conformal

(b) The combinatorial Yamabe flow will not develop essential singularities on any triangulation.

(c) The combinatorial Yamabe flow will converge to the constant curvature PL metric after finite number of surgeries on the triangulation.

There are some evidences indicating that the combinatorial Yamabe flow will develop removable singularities for some PL metrics on admissible triangulations. But we do not have the explicit examples yet.

It seems there is an interesting similarity between the singularity formation in combinatorial Yamabe flow on surfaces and Hamilton's Ricci flow program in dimension 3.

1.4. There are several interesting questions concerning the combinatorial Yamabe problem in higher dimension. To carry out the same program in higher dimension, the key ingredient that is missing is the following local rigidity property.

Question. Given an Euclidean n-simplex $\sigma_{n}$ and an (n-2)-face $\sigma_{n-2}$ of $\sigma_{n}$, let $a\left(\sigma_{n-2}, \sigma_{n}\right)$ be the dihedral angle of the n-simplex at the (n-2)-simplex. Now suppose $d: E \rightarrow \mathbf{R}_{>0}$ is the edge lengths of the $n$-simplex. Consider conformal factors $u: V \rightarrow \mathbf{R}_{>0}$ and the new PL metric $u * d$ on the n-simplex. Define the function $S_{u * d}: V \rightarrow \mathbf{R}^{n}$ to be $S_{u * d}(v)=\sum_{\sigma_{n-2}>v} a\left(\sigma_{n-2}, \sigma_{n}\right) \operatorname{vol}\left(\sigma_{n-2}\right)$ in the metric $u * d$. Then for fixed metric $d, S_{u * d}$ is a smooth function of $u$. Is the rank of the Jacobian matrix of the map $S_{u * d}$ considered as a function defined in an open set in $\mathbf{R}^{V}$ to $\mathbf{R}^{V}$ always equal to $n-1$ ?

An affirmative answer to this question for $n \geq 3$ will give a strong evidence that the higher dimensional combinatorial Yamabe flow exists, i.e., one would call equations of the type $\frac{d u_{i}}{d t}=f\left(u_{i}\right) S\left(v_{i}\right)$ the combinatorial Yamabe flow where $f(x)$ is some universal nowhere zero function depending on the dimension $n$. The function $f$ is so chosen that the corresponding matrix $\left[\frac{\partial S_{i}}{\partial u_{j}} f\left(u_{j}\right)\right]$ is symmetric. This question is also related to the following. Given a PL metric $d$ associated to a triangulation, consider the set of all conformal factors $u$ for $d$. Let $R(u * d)=\sum_{\sigma_{n-2}} K\left(\sigma_{n-2}\right) \operatorname{vol}\left(\sigma_{n-2}\right)$ be the Regge action of the metric $u * d$. Is the Hessian of $R(u * d)$ considered as a function of $u$ semi-positive definite?

The other related question we are considering now is the combinatorial Yamabe flow in hyperbolic or spherical background metric, i.e., we use hyperbolic or spherical simplexes instead of the Euclidean ones. The work of [CL] suggests that in general it is easier to work in hyperbolic geometry.

There are many interesting questions in the field of PL metric theory. For instance what is the right analog of the Ricci curvature? What is the Laplacian operator on the space of all functions defined on the $i$-simplexes? Is it true that positive PL scalar curvature carries topological information? To be more precise, suppose $M$ is a 3 -manifold which supports a positive PL scalar curvature. Is it ture that the fundamental group of $M$ 
contains no none-abelian surface group? In the smooth case, the result was proved by Schoen and Yau [SY].

1.5. In [CR], Cooper and Rivin define the concept of combinatorial scalar curvature differently. In their definition, the combinatorial scalar curvature is invariant under the scaling of metric. On the other hand, the scalar curvature in Riemannian geometry has the property that $S\left(k g_{i j}\right)=1 / k S\left(g_{i j}\right)$ for any positive constant k. (In our case, the PL scalar curvature satisfies $S(k g)=k^{n-2} S(g)$.) It can be shown every topological 3-manifold has a triangulation and an associated PL metric whose PL scalar curvature is positive in the sense of $[\mathrm{CR}]$. Thus the positivity of PL scalar curvature defined in the sense of [CR] does not carry any topological information. However, at each vertex, the scalar curvature defined in $[\mathrm{CR}]$ is a measurment of the infinitesimal rate of change of the volume of a small ball centered at the vertex. Thus, it is a combinatorial analogy of the scalar curvature in the infinittesimal sense. The work $[\mathrm{Ga}]$ follows the approach of $[\mathrm{CR}]$ and defines a combinatorial Yamabe flow for ball packing metrics. The PL scalar curvature defined in this paper is more closely related to Regge's calculus.

1.6. The paper is organized as follows. In $\S 2$, we establish some basic properties of the geometry of triangles. In $\S 3, \S 4$, and $\mathrm{S} 5$, we prove $1.1,1.2,1.3$, and 1.4 . In the appendix A, we provide a detail calculation involved in the proof of theorem 2.1. In appendix B, we use the feasible flow theorem for network flow to establish the necessity part of theorem 1.1.

1.6. This work is supported in part by the NSF. We thank Ben Chow, X-S. Lin and D. Sullivan for discussions. We thank the referee for making some nice suggestions.

\section{$\S 2$. Geometry of Euclidean Triangles}

For simplicity, let $\Delta=\left\{\left(x_{1}, x_{2}, x_{3}\right) \in \mathbf{R}_{>0}^{3} \mid x_{i}+x_{j}>x_{k}\right.$, where $\mathrm{i}, \mathrm{j}, \mathrm{k}$ are distinct $\}$ be the set of points whose coordinates satisfy the triangular inequalities. We use $\mathbf{R}_{>0}$ to denote the set of all positive numbers, and $\Delta v_{1} v_{2} v_{3}$ to denote the triangle having vertices $v_{1}, v_{2}$, and $v_{3}$. If $X$ is a finite set, we use $|X|$ to denote the number of elements in $X$.

2.1. Fix three numbers $d_{i}, d_{j}, d_{k}$ satisfying triangular inequalities, i.e., $\left(d_{i}, d_{j}, d_{k}\right) \in \Delta$ and three vertices $v_{i}, v_{j}, v_{k}$. Consider the conformal factor $u:\left\{v_{i}, v_{j}, v_{k}\right\} \rightarrow \mathbf{R}_{>0}$ so that $\left(d_{i} / u_{i}, d_{j} / u_{j}, d_{k} / u_{k}\right) \in \Delta$ where $u_{r}=u\left(v_{r}\right)$. Define $x_{r}=d_{r} u_{s} u_{t}$ where $\{r, s, t\}=\{i, j, k\}$. Due to the choice of $u_{r}$ 's, $\left(x_{i}, x_{j}, x_{k}\right) \in \Delta$. Construct a Euclidean triangle $\Delta v_{i} v_{j} v_{k}$ so that the length of the edge opposite to $v_{r}$ is $x_{r}$. Let $\theta_{r}$ be the inner angle at the vertex $v_{r}$. Then $\theta_{r}$ is a smooth function of $\left(u_{i}, u_{j}, u_{k}\right)$.

Theorem 2.1. The $3 \times 3$ matrix $\left[\frac{\partial \theta_{r}}{\partial u_{s}} u_{s}\right]_{3 \times 3}$ is symmetric, semi-negative definite and has rank 2 whose null space is $\left\{(t, t, t) \in \mathbf{R}^{3} \mid t \in \mathbf{R}\right\}$. Furthermore, if we let $a_{r r}=x_{r}^{2}$ and $a_{r s}=-x_{r} x_{s} \cos \left(\theta_{t}\right)$ where $\{r, s, t\}=\{i, j, k\}$ and let $A$ be the area of the triangle $\Delta v_{i} v_{j} v_{k}$, then $\left[\frac{\partial \theta_{r}}{\partial u_{s}} u_{s}\right]_{3 \times 3}=-\frac{1}{2 A}\left[a_{r s}\right]_{3 \times 3}$. 
The proof is a straight forward computation. We defer it to the appendix A. It is easy to see that the matrix $\left[a_{r s}\right]_{3 \times 3}$ is symmetric and semi-positive definite. Indeed, since $x_{i}=$ $x_{j} \cos \theta_{k}+x_{k} \cos \theta_{j}$, we see that the sum of entries in every row is zero. Thus $\operatorname{det}\left(\left[a_{r s}\right]_{3 \times 3}\right)=$ 0 . On the other hand, the diagonal entries are positive so are the determinants of the principal $2 \times 2$ submatrices. Thus the $3 \times 3$ matrix $\left[a_{r s}\right]_{3 \times 3}$ is semi-positive definite whose null space is the diagonal in $\mathbf{R}^{3}$.

Corollary 2.2. The matrix $\left[\frac{\partial \theta_{r}}{\partial u_{s}} u_{s}\right]_{3 \times 3}$ depends only on the three inner angles $\theta_{i}, \theta_{j}$ and $\theta_{k}$ of the triangle. In particular, if the conformal factor $u=\left(u_{i}, u_{j}, u_{k}\right)$ varies in a region so that the inner angles of the triangle $\Delta v_{i} v_{j} v_{k}$ lie in a compact set in the open interval $(0, \pi)$, then there is a positive constant $\lambda$ so that the negative eigenvalues of the matrix $\left[\frac{\partial \theta_{r}}{\partial u_{s}} u_{s}\right]_{3 \times 3}$ are less than $-\lambda$ for all $u$ in the region.

2.2. Using the same notations as above, let $w_{r}=\log u_{r}$ for $r=i, j, k$. Then the $3 \times 3$ matrix $\left[\frac{\partial \theta_{r}}{\partial w_{s}}\right]_{3 \times 3}$ is symmetric and semi-negative definite of rank 2 whose null space is $\left\{(t, t, t) \in \mathbf{R}^{3} \mid t \in \mathbf{R}\right\}$. Consider the non-convex space $W=\left\{w=\left(w_{i}, w_{j}, w_{k}\right) \in\right.$ $\left.\mathbf{R}^{3} \mid\left(d_{i} e^{-w_{i}}, d_{j} e^{-w_{j}}, d_{k} e^{-w_{k}}\right) \in \Delta\right\}$. The space $W$ is simply connected since it is the image of the convex space $\left\{\left(r_{i}, r_{j}, r_{k}\right) \in \mathbf{R}_{>0}^{3} \mid\left(d_{i} r_{i}, d_{j} r_{j}, d_{k} r_{k}\right) \in \Delta\right\}$ under the homeomorphism $h\left(r_{i}, r_{j}, r_{k}\right)=\left(-\log r_{i},-\log r_{j},-\log r_{k}\right)$. Furthermore, if $w \in W$, then $w+(t, t, t)$ is again in $W$ for any real number $t$. Take any vector $\left(a_{i}, a_{j}, a_{k}\right) \in \mathbf{R}$ and let $\Omega=\left(a_{i}-\theta_{i}\right) d w_{i}+$ $\left(a_{j}-\theta_{j}\right) d w_{j}+\left(a_{k}-\theta_{k}\right) d w_{k}$ be a smooth 1-form defined on the space $W$. It is closed due to the symmetry of the matrix $\left[\frac{\partial \theta_{r}}{\partial w_{s}}\right]_{3 \times 3}$. Define a smooth function $F(w): W \rightarrow R$ by

$$
F(w)=\int_{0}^{w} \Omega .
$$

This is well defined since the form $\Omega$ is closed and the space $W$ is simply connected.

Corollary 2.3. The function $F: W \rightarrow \mathbf{R}$ is a locally convex function so that it becomes a locally strictly convex function when restricted to the planes $\left\{\left(w_{i}, w_{j}, w_{k}\right) \in\right.$ $W \mid w_{i}+w_{j}+w_{k}$ is a constant $\}$. Furthermore,

(a). if $a_{i}+a_{j}+a_{k}=\pi$, then $F(w+(t, t, t))=F(w)$ for any real number $t$.

(b). if $\left(a_{i}, a_{j}, a_{k}\right) \in \mathbf{R}_{>0}^{3}$ and $a_{i}+a_{j}+a_{k}=\pi$, then for any sequence of points $w^{(n)}$ in $W$, $\lim \sup _{n \rightarrow \infty} F\left(w^{(n)}\right)=\infty$ if and only if $\limsup _{n \rightarrow \infty} \max _{\{r, s\} \subset\{i, j, k\}}\left(\left|w_{r}^{(n)}-w_{s}^{(n)}\right|\right)=$ $\infty$.

Proof. The local convexity is due to the fact the Hessian of $F$ is the matrix $-\left[\frac{\partial \theta_{r}}{\partial w_{s}}\right]_{3 \times 3}$ which is semi-positive definite. Furthermore, since the matrix is positive definite on the linear space $w_{i}+w_{j}+w_{k}=0$, it follows that $F$ is locally strictly convexity on the planes.

To see (a), it suffices to verify that $\int_{w}^{w+(d, d, d)} \Omega=0$. Now take the line segment $t w+(1-t)(d, d, d)$ in $W$ to evaluate the line integral $\int_{w}^{w+(d, d, d)} \Omega$. We find the integral becomes $\int_{0}^{1}\left(\left(a_{i}+a_{j}+a_{k}\right)-\left(\theta_{i}+\theta_{j}+\theta_{k}\right)\right) d t=0$ since the integrant is 0 .

To see (b), let $\Pi: \mathbf{R}^{3} \rightarrow\left\{\left(w_{1}, w_{2}, w_{3}\right) \in \mathbf{R}^{3} \mid w_{1}+w_{2}+w_{3}=0\right\}$ be the orthogonal projection. Then due to the condition $a i+a_{j}+a_{k}=\pi$ and part (a), we have $F(w)=$ 
$F(\Pi(w))$. Also, we have $\lim \sup _{n \rightarrow \infty} \max _{\{r, s\} \subset\{i, j, k\}}\left(\left|w_{r}^{(n)}-w_{s}^{(n)}\right|\right)=\infty$ if and only if their projections $\Pi\left(W^{(n)}\right)$ is unbounded. Thus if $\lim _{\sup } \rightarrow \infty \max _{\{r, s\} \subset\{i, j, k\}}\left(\left|w_{r}^{(n)}-w_{s}^{(n)}\right|\right)$ is finite, then the function $F\left(w^{n}\right)$ remains bounded.

Now suppose $\lim \sup _{n \rightarrow \infty} \max _{\{r, s\} \subset\{i, j, k\}}\left|w_{r}^{(n)}-w_{s}^{(n)}\right|=\infty$. By taking a subsequence if necessary, we may assume without loss of generality that $\lim _{n \rightarrow \infty}\left(w_{i}^{(n)}-w_{j}^{(n)}\right)=\infty$. By part (a), we may further assume after adding $w^{(n)}$ by a diagonal vector $\left(d_{n}, d_{n}, d_{n}\right)$ that $w^{(n)}$ is in the hypersurface $\left\{\left(w_{i}, w_{j}, w_{k}\right) \in W \mid e^{-w_{i}}+e^{-w_{j}}+e^{-w_{k}}=1\right\}$. In particular, $w_{r}^{(n)} \geq 0$. Thus, $\lim _{n \rightarrow \infty} w_{i}^{(n)}=\infty$. By the constraints $e^{-w_{i}}+e^{-w_{j}}+e^{-w_{k}}=1$ and the triangular inequalities $d_{i} e^{-w_{i}}+d_{r} e^{-w_{r}}>d_{s} e^{-w_{s}}$ for $\{r, s\}=\{j, k\}$, both $w_{j}^{(n)}$ and $w_{k}^{(n)}$ are bounded from above. This shows that the triangle $\Delta v_{i} v_{j} v_{k}$ with edge lengths $d_{i} e^{w_{j}^{(n)}+w_{k}^{(n)}}, d_{j} e^{w_{i}^{(n)}+w_{k}^{(n)}}$ and $d_{k} e^{w_{i}^{(n)}+w_{j}^{(n)}}$ degenerates into a half-line since two of the edge lengths tend to infinity and the third remains bounded. Thus $\theta_{i}\left(w^{(n)}\right)$ tends to zero. Therefore the dominate term in the integration $\int_{0}^{w^{(n)}} \Omega$ is $\int_{0}^{w_{i}^{(n)}}\left(a_{i}-\theta_{i}\right) d w_{i}$ since the other two integrals are bounded. Because $a_{i}>0$ and $\lim _{n} \theta_{i}\left(w^{(n)}\right)=0$, we see that $\lim _{n \rightarrow \infty} F\left(w^{(n)}\right)=\infty$. QED

2.3. Remarks. The situation in PL conformal geometry is very similar to the approach to circle packing by Colin de Verdiere in $[\mathrm{Cv}]$. The complication in the combinatorial Yamabe problem is caused by the fact that the space $W$ is not convex and the function $F: W \rightarrow \mathbf{R}$ is not proper.

\section{§3. A Proof of Theorem 1.1}

That the condition $(*)$ is sufficient was established in [CL] theorem 1.1 where we take the weight function $\phi=0$. Indeed, condition (1.3) for $\phi=0$ in theorem 1.1 in [CL] is exactly the condition $(*)$. Thus in this case, there is a very special type PL metric associated to the triangulation whose curvature at each vertex is $2 \pi \chi(M) /|V|$. The metric is obtained by assigning to each vertex $v_{i}$ a positive number $r_{i}$ and define the length at the edge $v_{i} v_{j}$ to be $r_{i}+r_{j}$.

To show that the condition is also necessary, we use the feasible flow theorem for network flow. Since the method of the proof is irrelevant to the rest of the paper, we defer the proof to appendix B.

The fact that the condition $(*)$ holds for all triangulations of surfaces of non-negative Euler characteristic was established in [CL].

\section{$\S 4$. Proofs of Theorems 1.2. and $\mathbf{1 . 3}$}

4.1. To derive the evolution of the curvature $K_{i}$ for the combinatorial Yamabe flow $\frac{d u_{i}}{d t}=-u_{i} K_{i}$, we note that the evolution of an individual inner angle, say $\theta_{i}^{j k}$ in a triangle $\Delta v_{i} v_{j} v_{k}$ at the vertex $v_{i}$, is the following.

$$
\frac{d \theta_{i}^{j k}}{d t}=\sum_{r=i, j, k} \frac{\partial \theta_{i}^{j k}}{\partial u_{r}} \frac{d u_{r}}{d t}
$$




$$
=-\sum_{r=i, j, k} \frac{\partial \theta_{i}^{j k}}{\partial u_{r}} u_{r} K_{r}
$$

Now the curvature $K_{i}$ at the i-th vertex is $2 \pi-\sum_{j, k} \theta_{i}^{j k}$ where the summation is over all triangle $\Delta v_{i} v_{j} v_{k}$ in $T$. Thus $\frac{d K_{i}}{d t}=-\sum_{j, k} \frac{d \theta_{i}^{j k}}{d t}$. By (4.1) and theorem 2.1, we see that the curvature evolution equation is

$$
\frac{d K_{i}}{d t}=\sum_{r=1}^{N} \sum_{j, k}^{N} \frac{\partial \theta_{i}^{j k}}{\partial u_{r}} u_{r} K_{r}=\sum_{r=1}^{N} c_{i r} K_{r} .
$$

We can write the coefficient matrix as a sum of matrices,

$$
\left[c_{i r}\right]_{N \times N}=\sum_{j, k}\left[\frac{\partial \theta_{i}^{j k}}{\partial u_{r}} u_{r}\right]_{N \times N}
$$

where the sum is over all ordered edges $\{j, k\}$. The matrix $A^{j, k}=\left[\frac{\partial \theta_{i}^{j k}}{\partial u_{r}} u_{r}\right]_{N \times N}$ is symmetric and semi-positive definite with only one $3 \times 3$ submatrix being non-zero and all other entries being zero by theorem 2.1. Thus the matrix $\left[c_{i j}\right]$ is symmetric and semi-positive definite. Furthermore, by the same identity above and theorem 2.1, we see that the rank of $\left[c_{i j}\right]$ is $N-1$ whose null space is spanned by the vector $[1,1, \ldots, 1]$.

Take the time derivative of $G(t)=\sum_{i=1}^{N} K_{i}^{2}(t)$. We get

$$
\frac{d G}{d t}=2 \sum_{i=1} K_{i} K_{i}^{\prime}=2 \sum_{i, j=1}^{N} c_{i j} K_{i} K_{j} \leq 0
$$

Thus the total curvature square is decreasing along the solution. This establishes part (a) of theorem 1.2.

4.2. To show part (b), if we change the variable $u_{i}$ to $w_{i}=\log u_{i}$, then the combinatorial Yamabe flow becomes $\frac{d w_{i}}{d t}=-K_{i}$. Note that the symmetry of the matrix $\left[\frac{\partial \theta_{r}}{\partial u_{s}} u_{s}\right]_{3 \times 3}$ implies that the $N \times N$ matrix $\left[\frac{\partial K_{r}}{\partial w_{s}}\right]_{N \times N}$ is symmetric and is semi-positive definite. Its rank is $(\mathrm{N}-1)$ and its null space is $\{(t, t, \ldots, t) \mid t \in \mathbf{R}\}$.

Define $\mathcal{W}$ to be the space $\left\{w=\left(w_{1}, \ldots, w_{N}\right) \in \mathbf{R}^{N} \mid\right.$ if $v_{i} v_{j} v_{k}$ forms a triangle in $T$, then $\left.\left(w_{i}, w_{j}, w_{k}\right) \in W\right\}$. Note that $\mathcal{W}$ is not a convex space but is simply connected since it is the image of the convex set $\left\{\left(r_{1}, \ldots, r_{N}\right) \in \mathbf{R}_{>0}^{N} \mid\right.$ if $v_{i} v_{j} v_{k}$ forms a triangle in $T$, then $\left.d_{i} r_{i}+d_{j} r_{j}>d_{k} r_{k}\right\}$ under the diffeomorphism $\left(r_{1}, \ldots, r_{N}\right) \rightarrow\left(-\log r_{1}, \ldots,-\log r_{N}\right)$. Also, if $w \in \mathcal{W}$, then $w+(t, t, \ldots, t)$ is still in $\mathcal{W}$.

Define a 1-form $\Omega=\sum_{i=1}^{N} K_{i} d w_{i}$ on the space $\mathcal{W}$. This form is closed since the matrix $\left[\frac{\partial K_{r}}{\partial w_{s}}\right]_{N \times N}$ is symmetric. Thus the function

$$
F(w)=\int_{0}^{w} \Omega
$$


is well defined on the simply connected space $\mathcal{W}$. The function $F$ is locally convex since its Hessian is the semi-positive definite matrix $\left[\frac{\partial K_{r}}{\partial w_{s}}\right]_{N \times N}$ and is locally strictly convex on the planes $\mathcal{W} \cap\left\{w \mid w_{1}+\ldots+w_{N}=\right.$ constant $\}$ since the matrix is positive definite when restricted to the plane $\left\{w \mid w_{1}+\ldots+w_{N}=0\right\}$. Due to $\frac{\partial F}{w_{i}}=K_{i}$, it follows that the combinatorial Yamabe flow $\frac{d w_{i}}{d t}=-K_{i}$ is the negative gradient flow of a locally convex function defined on $\mathcal{W}$.

4.3. To see the local rigidity, we note that under the transformation from $u$ to $w$, the space $u$-space becomes the (N-1)-dimensional smooth manifold $P=\mathcal{W} \cap\left\{w_{1}+\ldots+w_{N}=0\right\}$. The curvature map $K$ becomes the map sending $w \in P$ to the gradient of a locally strictly convex function $\left.F\right|_{P}$ defined on $P$. Due to the local strictly convexity, the gradient map is locally injective. Since the image of $K$ is $\left\{\left(k_{1}, \ldots, k_{N}\right) \mid \sum_{i=1}^{N} k_{i}=2 \pi \chi(M)\right\}$ which is also a manifold of dimension (N-1), by the invariance of domain theorem, the curvature map $K$ must be a local homeomorphism. This establishes part (c).

4.4. Remark. It can be shown that the curvature map $K$ is a local diffeomorphism. However, we are not able to show that is globally a diffeomorphism.

4.5. To prove part (a) of theorem 1.3, note that if $u_{i}(t)$ solves the combinatorial Yamabe flow, then due to the universal curvature bound that $(2-|E|) \pi \leq K_{i}<2 \pi$, we have

$$
1 / c e^{-c t} \leq u_{i}(t) \leq c e^{c t}
$$

for some positive constant $c$ on the time interval $[0, L)$ where the solution exists. Thus no essential singularity develops on any triangulation in finite time.

4.6. To prove part (b) of theorem 1.3, since the triangulation is admissible, we find a PL flat metric associated to the triangulation so that its curvature is constant $2 \pi \chi(M) / N$. Let $a_{i}^{j k}$ be the inner angle at vertex $v_{i}$ in triangle $\Delta v_{i} v_{j} v_{k}$ in the metric. For the normalized equation $u_{i}^{\prime}=-u_{i}\left(K_{i}-K_{a v}\right)$, we make a change of variable $w_{i}=\log u_{i}$ as before. By the normalization condition that $\prod_{i=1} u_{i}(t)=1$ for all time, we have $\sum_{i=1}^{n} w_{i}(t)=0$. By the same calculation, we still have the fact that the $N \times N$ matrix $\left[\frac{\partial\left(K_{r}-K_{a v}\right)}{\partial w_{s}}\right]_{N \times N}$ is again symmetric, semi-positive definite of rank (N-1) whose null space is $\left\{(t, t, \ldots, t) \in \mathbf{R}^{N} \mid t \in\right.$ R\}.

Now consider the space $\mathcal{W}$ as in subsection 4.2. We construct a specific 1 -form $\Omega$ as

$$
\Omega=\sum_{i=1}^{N}\left(\sum_{j, k}\left(a_{i}^{j k}-\theta_{i}^{j k}\right) d w_{i}\right)
$$

where $\theta_{i}^{j k}$ is the inner angle of the metric $u * d$ at the vertex $v_{i}$ inside the triangle $\Delta v_{i} v_{j} v_{k}$. By the choice of the inner angles $a_{i}^{j k}$, the 1 -form $\Omega$ is exactly $\sum_{i=1}^{N}\left(K_{i}-K_{a v}\right) d w_{i}$.

Let us now consider the associated function $F(w)=\int_{0}^{w} \Omega$ defined on $\mathcal{W}$. This function is well defined since $\mathcal{W}$ is simply connected and $\Omega$ is closed. Furthermore, by the construction, the combinatorial Yamabe flow $w_{i}^{\prime}=-\left(K_{i}-K_{a v}\right)$ is the negative gradient 
flow of $F(w)$. In particular, if $w(t)$ is a solution to the combinatorial Yamabe flow, the function $h(t)=F(w(t))$ is decreasing in time $t$.

On the other and, we can rewrite the summation in (4.2) as follows,

$$
F(w)=\sum_{\Delta v_{i} v_{j} v_{k} \in T} \int_{0}^{w}\left(a_{i}^{j k}-\theta_{i}^{j k}\right) d w_{i}+\left(a_{j}^{i k}-\theta_{j}^{i k}\right) d w_{j}+\left(a_{k}^{i j}-\theta_{k}^{i j}\right) d w_{k}
$$

where the sum is over all triangles in $T$.

Since $a_{i}^{j k}+a_{j}^{k i}+a_{k}^{i j}=\pi$ over each triangle, by corollary $2.3(\mathrm{a}), F(w+(d, \ldots, d))=F(w)$ for all $w \in \mathcal{W}$. Now suppose the normalized combinatorial Yamabe flow $w_{i}^{\prime}=-\left(K_{i}-K_{a v}\right)$ develops an essential singularity at time $t=L \leq \infty$. Then, due to the normalization equation $\sum_{i=1}^{N} w_{i}(t)=0$, there is a sequence of time $t_{n} \rightarrow L$ and two indices $i, j$ so that $\lim _{t_{n} \rightarrow \infty} w_{i}\left(t_{n}\right)=\infty$ and $\lim _{t_{n} \rightarrow \infty} w_{j}\left(t_{n}\right)=-\infty$.

We claim in this case $\lim _{t_{n} \rightarrow L} F\left(w\left(t_{n}\right)\right)=\infty$. This will contradict the fact that $F(w(t))$ is decreasing in time $t$.

To see that claim, we use the same argument used in the proof of corollary 2.3(b). Indeed, after adding $w\left(t_{n}\right)$ by a vector of the form $\left(d_{n}, \ldots d_{n}\right)$, we may assume that $w\left(t_{n}\right)$ is in the subspace $\left\{\left(w_{1}, \ldots, w_{N}\right) \in \mathbf{R}^{N} \mid e^{-w_{1}}+e^{-w_{2}}+\ldots+e^{-w_{N}}=1\right\}$. In this case, we have $w_{j}\left(t_{n}\right) \geq 0$ for all $j$. Also there are two indices $i, j$ so that

$$
\lim _{t_{n} \rightarrow L} w_{i}\left(t_{n}\right)=\infty \text { and } w_{j}\left(t_{n}\right) \text { remains bounded. }
$$

Now by (4.3) the integral $F\left(w\left(t_{n}\right)\right)$ is the sum of finitely many integrals of type (2.1) over each triangle $\Delta v_{i} v_{j} v_{k}$ where the vector $\left(a_{i}^{j k}, a_{j}^{i k}, a_{k}^{i j}\right)$ satisfies the condition in corollary 2.3(b). Thus, by the same argument used in the proof of corollary 2.3(b), the

integral $\int_{0}^{w\left(t_{n}\right)}\left(a_{i}^{j k}-\theta_{i}^{j k}\right) d w_{i}+\left(a_{j}^{i k}-\theta_{j}^{i k}\right) d w_{j}+\left(a_{k}^{i j}-\theta_{k}^{i j}\right) d w_{k}$ is either bounded or tends to infinity depending on $\lim \sup _{n \rightarrow \infty} \max _{\{r, s\} \subset\{i, j, k\}}\left(\left|w_{r}\left(t_{n}\right)-w_{s}\left(t_{n}\right)\right|\right)$ is finite or infinite. However, the infinite case must occur due to (4.4). This shows that $F\left(w\left(t_{n}\right)\right)$ tends to infinity. QED

\section{$\S 5$. A Proof of theorem 1.4.}

By the assumption, the solution $u(t)=\left(u_{1}(t), \ldots, u_{N}(t)\right)$ of the normalized combinatorial Yamabe flow exists for all time so that there are no singularities forming at time equal to infinity. This means that $u_{i}(t)$ 's are in some compact interval in $\mathbf{R}_{>0}$ and also all inner angles $\theta_{i}^{i j}(t)$ are in some compact interval inside the interval $(0, \pi)$. By corollary 2.2 , this implies that the there is a positive constant $\lambda$ so that the eigenvalues of coefficient matrix $\left[c_{r s}\right]_{N \times N}$ considered as a bilinear form restricted to the subspace $\left\{w \in \mathbf{R}^{N} \mid w_{1}+\ldots+w_{N}=0\right\}$ is always bounded by $-\lambda$ for all time $t \in[0, \infty)$, i.e.,

$$
\sum_{i, j} c_{i j} w_{i} w_{j} \leq-\lambda \sum_{i} w_{i}^{2}, \quad \text { when } \sum_{i=1}^{N} w_{i}=0
$$


To prove the theorem, it suffices to show that the curvature $K_{i}(t)-K_{a v}$ converges to 0 exponentially fast, i.e., there is a positive constants $c_{1}, c_{2}$ so that

$$
\left|K_{i}(t)-K_{a v}\right| \leq c_{1} e^{-c_{2} t}
$$

Assuming (5.2) holds, then we can solve $u_{i}(t)$ from the combinatorial Yamabe flow and get

$$
u_{i}(t)=e^{-\int_{0}^{t}\left(K_{i}(s)-K_{a v}\right) d s} .
$$

This shows that $\lim _{t \rightarrow \infty} u_{i}(t)$ is a positive real number for all indices. Thus the metrics $u(t) d$ converges to the constant PL curvature metric.

To establish (5.1), let us consider the function

$$
G(t)=\sum_{i=1}^{N}\left(K_{i}(t)-K_{a v}\right)^{2}
$$

Its derivative can be calculated as

$$
G^{\prime}(t)=2 \sum_{i, j} c_{i j}\left(K_{i}-K_{a v}\right)\left(K_{j}-K_{a v}\right)
$$

By (5.1), we have $G^{\prime}(t) \leq-\lambda G(t)$. Thus $G(t) \leq C e^{-\lambda t}$. This establishes (5.2). QED

5.2. Remark. One can now show that the normalized combinatorial Yamabe flow for a single Euclidean triangle $\frac{d u_{i}}{d t}=-\left(\pi / 3-\theta_{i}\right) u_{i}$ converges to the equilateral triangle as follows. By the same argument as in the proof of theorem 1.3, we see that essential singularity never occur. If a removable singularity occurs at time $t=L$, then the three inner angles tend to $0,0, \pi$. On the other hand the sum of the square of the curvature $\sum_{r}\left(K_{i}-K_{a v}\right)^{2}$ achieves its supremum value only when the inner angles are $0,0, \pi$. Thus by theorem 1.2(a) that the sum of the square of the curvature is decreasing, removable singularities never occur. By theorem 1.4, we see the solution converges. This ends the proof.

\section{Reference}

[A] Aubin, T., quations diffrentielles non linaires et problme de Yamabe concernant la courbure scalaire. J. Math. Pures Appl. (9) 55 (1976), no. 3, 269-296.

[CE] Cao, J.-G.; Escobar, J. F., A New 3-dimensional Curvature Integral Formula for PL-manifolds of Non-positive Curvature, preprint, Feb. 2000.

[CL] Chow, B., and Luo, F., Combinatorial Ricci flows on surfaces, preprint, 2002, http://front.math.ucdavis.edu/math.DG/0211256.

[CMS] Cheeger, J.; Müller, W.; Schrader, R., On the curvature of piecewise flat spaces. Comm. Math. Phys. 92 (1984), no. 3, 405-454. 
[CR] Cooper, D.; Rivin, I., Combinatorial scalar curvature and rigidity of ball packings. Math. Res. Lett. 3 (1996), no. 1, 51-60.

[Cv] Colin de Verdire, Y., Un principe variationnel pour les empilements de cercles. Invent. Math. 104 (1991), no. 3, 655-669.

[FF] Ford, L. R., Jr.; Fulkerson, D. R., Flows in networks. Princeton University Press, Princeton, N.J. 1962

[Ga] Glickenstein, D., A maximum principle for combinatorial Yamabe flow, preprint, 2002.

[Re] Regge, T., General relativity without coordinates, Nuovo Cimento, 19,(1961),558571.

[Sc] Schoen, R., Conformal deformation of a Riemannian metric to constant scalar curvature. J. Differential Geom. 20 (1984), no. 2, 479-495.

[St] Stone, D. A., Geodesics in piecewise linear manifolds. Trans. Amer. Math. Soc. 215 (1976), 1-44.

[SY] Schoen, R.; Yau, S. T., Existence of incompressible minimal surfaces and the topology of three-dimensional manifolds with nonnegative scalar curvature. Ann. of Math. (2) 110 (1979), no. 1, 127-142.

[Tu] Trudinger, N. S., Remarks concerning the conformal deformation of Riemannian structures on compact manifolds. Ann. Scuola Norm. Sup. Pisa (3) 221968 265-274.

[Ya] Yamabe, H., On a deformation of Riemannian structures on compact manifolds. Osaka Math. J. 121960 21-37.

\section{Appendix A. A Proof of Theorem 2.1}

We will carry out the computational aspect of the proof of theorem 2.1.

The following lemma was established in [CL], Lemma A-1.

Lemma A-1. Suppose $\Delta v_{i} v_{j} v_{k}$ is a Euclidean triangle of area $A$ so that the inner angle at $v_{i}$ is $\theta_{i}$ and the length of the edge $v_{j} v_{k}$ is $x_{i}$. Then $\theta_{i}$ is a smooth function of $\left(x_{i}, x_{j}, x_{k}\right)$. The partial derivatives of the function are given by,

(a) $\frac{\partial \theta_{i}}{\partial x_{i}}=x_{i} /(2 A)$.

(b) $\frac{\partial \theta_{i}}{\partial x_{j}}=-\frac{\partial \theta_{i}}{\partial x_{i}} \cos \left(\theta_{k}\right)$.

In our case, we fix a set of positive numbers $d_{i}, d_{j}, d_{k}$ and choose a conformal factor $\left(u_{i}, u_{j}, u_{k}\right) \in \mathbf{R}_{>0}^{3}$. The edge lengths of the triangle are $x_{i}=d_{i} u_{j} u_{k}$. Thus $\frac{\partial x_{i}}{\partial u_{i}}=0$ and $\frac{\partial x_{i}}{\partial u_{j}}=x_{i} / u_{j}$.

Now the partial derivative can be calculated by the chain rule,

$$
\begin{gathered}
\frac{\partial \theta_{i}}{\partial u_{r}}=\sum_{s} \frac{\partial \theta_{i}}{\partial x_{s}} \frac{\partial x_{s}}{\partial u_{r}} \\
=\sum_{s \neq r} \frac{\partial \theta_{i}}{\partial x_{s}} \frac{x_{s}}{u_{r}}
\end{gathered}
$$


This shows that $\frac{\partial \theta_{i}}{\partial u_{r}} u_{r}=\sum_{s \neq r} \frac{\partial \theta_{i}}{\partial x_{s}} x_{s}$. Now use the lemma A-1 above together with the fact that $x_{i}=x_{j} \cos \theta_{k}+x_{k} \cos \theta_{j}$, we obtain $\frac{\partial \theta_{i}}{\partial u_{r}} u_{r}=-\frac{a_{i r}}{2 A}$ as stated in theorem 2.1.

\section{Appendix B. An Application of the Feasible Flow Theorem to Theorem 1.1}

We now verify that the condition $(*)$ in theorem 1.1 is also sufficient for the existence of constant curvature PL metric associated to the triangulation. This proof follows the same ideas appeared in $[\mathrm{Cv}]$.

Let us begin with the feasible flow theorem for network flow. Suppose $G=(V, E)$ is a directed graph, i.e., a graph so that each edge is oriented. Here $V$ is the set of all vertices and $E$ is the set of all oriented edges. For each oriented edge $x \in E$, let $\operatorname{int}(x)$ and $\operatorname{end}(x)$ be the initial vertex and the end vertex of the edge $x$. (It is possible that $\operatorname{int}(x)=\operatorname{end}(x)$.) For any subset $I \subset V$, let $\operatorname{int}(I)=\{x \in E \mid \operatorname{int}(x) \in I$, end $(x) \notin I\}$, and let $\operatorname{end}(I)=\{x \in E \mid \operatorname{end}(x) \in I, \operatorname{int}(x) \notin I\}$.

Assume there is a lower capacity bound $a: E \rightarrow[-\infty, \infty]$ and an upper capacity bound $b: E \rightarrow[-\infty, \infty]$ so that $a(x) \leq b(x)$ for all $x \in E$.

A feasible flow on the graph $G$ is a function $\phi: E \rightarrow(-\infty, \infty)$ so that Kirchoff's current law is satisfied, i.e., for each vertex $v$

$$
\sum_{x \in \operatorname{end}(v)} \phi(x)=\sum_{x \in \operatorname{int}(v)} \phi(x)
$$

and $a(x) \leq \phi(x) \leq b(x)$ for all $x \in E$.

Feasible Flow Theorem. A feasible flow exists if and only if for every non-empty subset $U \subset V$ so that $U \neq V$,

$$
\sum_{x \in \operatorname{end}(U)} b(x) \geq \sum_{x \in \operatorname{int}(U)} a(x)
$$

We now apply the theorem to show that condition $(*)$ in theorem 1.1 is a necessary condition. To this end, let the set of all vertices in the triangulation be $V$ and the set of all triangle in $T$ be $F$. If $v \in V$ and $f \in F$, then $f>v$ means $v$ is a vertex of $f$. Let $z$ be an extra vertex. Define an oriented graph $G$ as follows. The set of all vertices in $G$, denoted by $G^{0}$ is $V \cup F \cup\{z\}$. The set of all oriented edges $G^{1}$ is $\{(f, v) \mid f \in F, v \in V, f>$ $v\} \cup\{(z, f) \mid f \in F\} \cup\{(v, z) \mid v \in V\}$.

Now suppose there is a constant PL flat metric associated to the triangulation. Define a feasible flow $\phi: G^{1} \rightarrow[0, \infty)$ on the graph $G$ as follows: $\phi((f, v))$ is the inner angle of the triangle $f$ at the vertex $v$, and $\phi((z, f))=\pi$ and $\phi((v, z))=\pi|F| /|V|$. Define the lower capacity $a$ for $G$ as follows: $a((f, v))=\epsilon>0$ which is smaller than any of the inner angles, $a(x)=\phi(x)$ for all other edges. Define the upper capacity $b$ for $G$ as follows: $b((f, v))=\infty$, $b(x)=a(x)$ for all other edges.

To verify the Kirchoff's law for $\phi$, it suffices to check the following three statements. At each vertex $v \in V$, it states that the sum of the inner angles at the vertex is $2 \pi-$ 
$2 \pi(\chi(M) /|V|)=\pi|F| /|V|$, i.e., the curvature is constant. At each vertex $f \in F$, it states that the sum of the inner angles of a triangle is $\pi$, and at the vertex $z$, it is the trivial statement that $\pi|F|=|V|(\pi|F| /|V|)$.

Recall that for a non-empty subset $I$ of $V$ so that $I \neq V$, we use $F_{I}=\{f \in F \mid f>v$ for some $v \in I\}$. For this set $I$, we consider the proper subset $U=I \cup F_{I}$ of vertices $G^{0}$. We claim that the feasibility condition (B1) for this $U$ is exactly the condition (*) in theorem 1.1.

Indeed, we have $\operatorname{end}(U)=\left\{(f, v) \mid v \in I, f \notin F_{I}, f>v\right\} \cup\left\{(z, f) \mid f \in F_{I}\right\}$. Note that by the choice of $F_{I}$, the set $\left\{(f, v) \mid v \in I, f \notin F_{I}, f>v\right\}=\emptyset$. Thus $\operatorname{end}(U)=\left\{(z, f) \mid f \in F_{I}\right\}$. Also, we have $\operatorname{int}(U)=\{(v, z) \mid v \in I\} \cup\left\{(f, v) \mid f \in F_{I}, v \notin I, f>v\right\}$. Thus the feasibility condition states that

$$
\sum_{f \in F_{I}} \pi \geq \sum_{v \in I} \pi(|F| /|V|)+\sum_{\left\{(f, v) \mid f \in F_{I}, v \notin I, f>v\right\}} \epsilon>\sum_{v \in I} \pi(|F| /|V|) .
$$

Thus we have $\left|F_{I}\right| /|I|>|F| /|V|$.

It can be shown easily that the condition $(*)$ in theorem 1.1 is exactly the feasibility condition in the feasible flow theorem. Thus one can in fact avoid using the result from [CL] in the proof of theorem 1.3. Namely, we choose the set of inner angles $a_{i}^{j k}$ in the proof by applying the feasible flow theorem on the same graph $G$ and the same upper and lower capacities $a$ and $b$.

Department of Mathematics

Rutgers University

New Brunswick, NJ 08845

email: fluo@math.rutgers.edu 